\title{
EFFECT OF NON-UNIFORM TORSION ON ELASTOSTATICS OF A FRAME OF HOLLOW RECTANGULAR CROSS-SECTION
}

\author{
MURÍN Justín ${ }^{1}$, AMINBAGHAI Mehdi², GOGA Vladimír1 ${ }^{1}$, KUTIŠ Vladimír ${ }^{1}$, \\ PAULECH Juraj ${ }^{1}$, HRABOVSKÝ Juraj ${ }^{1}$
}

\author{
${ }^{I}$ Slovak University of Technology in Bratislava, Faculty of Electrical Engineering and Information Technology, \\ Department of Applied Mechanics and Mechatronics, IPEAE, Ilkovičova 3, 81219 Bratislava, Slovak Republic, \\ email: justin.murin@stuba.sk \\ ${ }^{2}$ Vienna University of Technology, Institute for Mechanics of Materials and Structures, Karlsplatz 13, \\ A-1040 Vienna, Austria
}

\begin{abstract}
In this paper, results of numerical simulations and measurements are presented concerning the nonuniform torsion and bending of an angled members of hollow cross-section. In numerical simulation, our linearelastic 3D Timoshenko warping beam finite element is used, which allows consideration of non-uniform torsion. The finite element is suitable for analysis of spatial structures consisting of beams with constant open and closed cross-sections. The effect of the secondary torsional moment and of the shear forces on the deformation is included in the local finite beam element stiffness matrix. The warping part of the first derivative of the twist angle due to bimoment is considered as an additional degree of freedom at the nodes of the finite elements. Standard beam, shell and solid finite elements are also used in the comparative stress and deformation simulations. Results of the numerical experiments are discussed, compared, and evaluated. Measurements are performed for confirmation of the calculated results.
\end{abstract}

KEYWORDS: Non-uniform torsion, Angled frame, Hollow rectangular cross-section, Measurements and numerical calculations

\section{Introduction}

The effect of non-uniform torsion must be considered in the structural elastostatic and elastodynamic analyses of straight and curved thin-walled beams with both open and closed cross-sections. The maximum normal stress due to the bimoment occurs at the points action of the external torques (except for a free end of the beam) and at the cross-sections of restrained warping (e.g. clamped cross-sections). Special theories of torsion with warping, usually referred to as non-uniform torsion or warping torsion, were used to solve such problems analytically (e.g. [1]). The analogy between the 2nd-order beam theory with axial tension and torsion including warping has also often been exploited (e.g. [2], [3]). However, it is worth of note that in the literature and in engineering practice, as well as in the guidelines provided by Eurocode 3 [4], the significance of the effect of warping is assumed to be restricted to open cross-sections. Warping-based stresses and deformations in closed cross-sections are assumed to be insignificant and have, therefore, generally been neglected.

According to the aforementioned theory of torsion of open cross-sections including warping and according to the mentioned analogy, special finite beam elements were designed and implemented into finite element codes (e.g. [5], [6]). The warping effect was included through an additional degree of freedom at each nodal point in form of the first derivative of the angle of twist of the cross-section of the beam. Comprehensive overview of the literature dealing with the issue of a non-uniform torsion in elastostatics and elastodynamics of thin-walled beams 
can be found in the articles [7,8], for example. The actual research results show that for nonuniform torsion of beams with closed cross-sections the impact of the Secondary Torsion Moment Deformation Effect (STMDE) is especially significant [3, 9]. It is also shown [10, 11], that not only warping, but also a cross-section distortion plays very important role. Consideration of this effect leads to developing of new approaches with definition of more than 7 degrees of freedom in the beam cross-section, what an usual case is. If the new degrees of freedom not a tensor quantities are, then a problem arises in their transformation from a local to a global coordinate system, for example in the case of angled beam structures. However, it should be noted that taking into account the cross-sectional distortion can greatly reduce the disproportion between the results of the non-uniform torsion solution with finite beam elements on the one side and the solids respectively shell finite elements on the other side. As shown for warping modal analysis [9, 12], the acceptable eigenfrequencies match was achieved for the open cross-sections beams, and also for the first eigenfrequency of the hollow cross-section beams. Higher eigenforms of the hollow cross-section are characterized by a pronounced crosssectional distortion that reduces the torsional stiffness of the cross section over that which is calculated in the sense of Thin Tube Theory (TTT). But as shown in [7, 13], the results of an elastostatic analysis of the thin-walled straight beams with our warping beam finite element agree well with the ones obtained with the solid finite elements [7, 13].

In the actual research papers, there is very hard to find contributions dealing with elastostatics or elastodynamics of thin-walled angled frames including non-uniform torsion. One interesting description of this problem is made in the PhD work [14] where the effect of warping and distortion is shown on deformation of an angled members of hollow rectangular cross-section under out-of plane loading. Assessment of the 32x32 stiffness matrix of new beam element with joint modelling is presented. A very good agreement of obtained displacements with the ones obtained with finite shell elements is presented. The results comparison has also shown significant difference to the results obtained with TTT beam elements and by Timoshenko beam finite elements.

In this paper, results of bending, uniform and non-uniform torsion elastostatic analysis of the angled frame, consisted of rectangular hollow cross-section beams, are presented.

In chapter 2, a brief summary of our 3D-beam finite element with warping torsion (WT) is presented with $14 \times 14$ stiffness matrix [7]. In non-uniform torsion, the part of the bicurvature caused by the bimoment is taken into account as the warping degree of freedom, and the STMDE is also considered.

Chapter 3 contains the numerical and analytical investigations. The results from elastostatic analysis of the angled member of rectangular hollow cross-sections with WT beam finite element are presented and compared with the ones obtained by commercial FEM codes and an analytical method. Measurements are performed for confirmation of some of the calculated results. The final assessment of the obtained results is contained in the conclusions.

The main novelty of this paper is to extension of the knowledge of article [7] to elastostatic analysis of the angled frames with hollow cross-section, to show the effects of non-uniform torsion, and performing comparative analysis by the finite element method using standard beam, solid and shell elements. Numerical results are validated with the measurements.

\section{Finite element equations of Timoshenko 3D beam finite element (WT) including torsional warping and STMDE}

\subsection{Local finite element equations}


Fig. 1 shows a doubly symmetric prismatic beam element of length $L^{e}$, with the two nodes $i$ and $k$, and with appropriate geometric, material, static and kinematic parameters: $A$ is the cross-sectional area; $I_{y}$ and $I_{z}$ are the quadratic area moments of inertia; $I_{T}$ is the torsion constant; $I_{\omega}$ is the warping constant; $I_{T S}$ is the secondary torsion constant. The material properties are Young's modulus $E$ and the shear modulus $G$. In order to include warping, an additional degree of freedom is added to the classical nodal variables at each element nodal point. As mentioned previously, the warping part of the first derivative of the twist angle, $\vartheta_{M}^{\prime}$, is considered as this degree of freedom [7]. This is advantageous for the formulation of boundary conditions. If the effect of the secondary torsional moment on the deformations is not considered, $\vartheta_{M}^{\prime}(x) \equiv \vartheta^{\prime}(x)$. Here, $\vartheta^{\prime}(x)$ is the first derivative of the angle of twist (bicurvature) that is very often used as a 7 th degree of freedom in the usual non-uniform torsion formulations [1], [5], [6].

The nodal displacement vector in the local coordinate system, as shown in Fig. 1, is given as

$$
\left\{u^{e}\right\}^{T}=\left\{\begin{array}{llllllllllllll}
u_{i} & v_{i} & w_{i} & \vartheta_{x i} & \vartheta_{y i} & \vartheta_{z i} & \vartheta_{M i}^{\prime} & u_{k} & v_{k} & w_{k} & \vartheta_{x k} & \vartheta_{y k} & \vartheta_{z k} & \vartheta_{M k}^{\prime}
\end{array}\right\}
$$

where $u, v, w$ and $\vartheta_{x}, \vartheta_{y}, \vartheta_{z}$ are the classical degrees of freedom at the nodal points $i$ and $k$. As mentioned above, the 7 th degree of freedom at each node is a part of the bicurvature caused by the bimoment $M_{\omega}: \vartheta_{M i}^{\prime}$ and $\vartheta_{M k}^{\prime}$. The respective nodal loads vector is given as

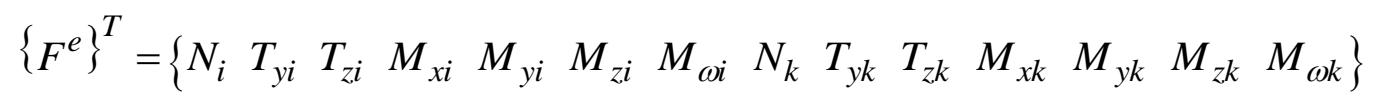

where $M_{x i}=M_{T i}$ and $M_{x k}=M_{T k}$ are the torsional moments, $M_{\omega i}$ and $M_{\omega k}$ are the bimoments, $M_{y i}, M_{y k}, M_{z i}, M_{z k}$ are the bending moments, $N_{i}$ and $N_{k}$ are the axial forces, and $T_{y i}, T_{y k}, T_{z i}, T_{z k}$ are the shear forces.

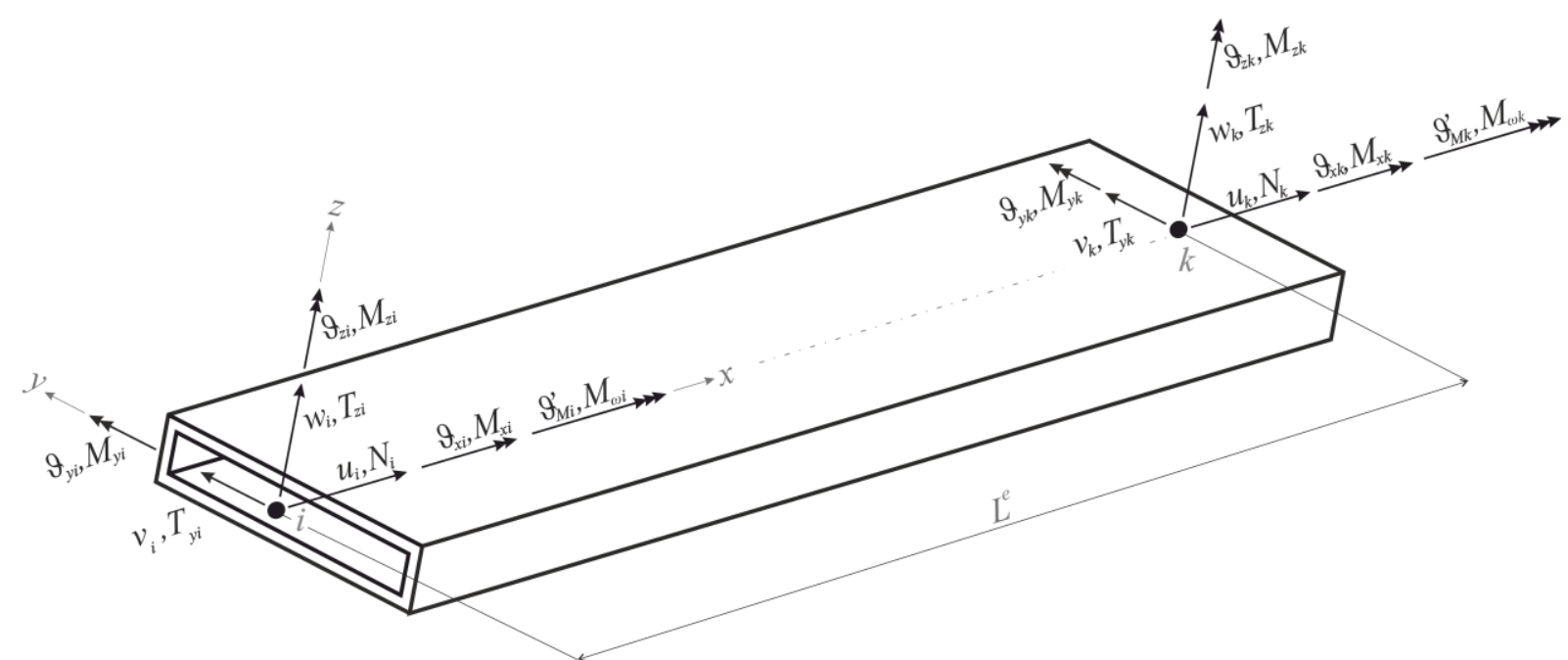

Fig. 1 WT-beam finite element, considering non-uniform torsion, embedded in the local coordinate system.

Enhancing the classical representation of Timoshenko finite beam elements by the stiffness matrix for non-uniform torsion of straight beams [7], gives the local equations for our finite Timoshenko beam finite element (WT-beam) considering warping: 


$$
\left\{F^{e}\right\}=\left[K^{l e}\right]\left\{u^{e}\right\}
$$

The effect of the secondary torsional moment and of the shear force on the deformations is included in the local finite element stiffness matrix $\left[K^{l e}\right]$, relating the nodal displacements $\left\{u^{e}\right\}$ to the nodal forces $\left\{F^{e}\right\} .\left[K^{l e}\right]$ is given as:

$$
\left[K^{l e}\right]=\left[\begin{array}{cccccccccccccc}
k_{1,1} & 0 & 0 & 0 & 0 & 0 & 0 & k_{1,8} & 0 & 0 & 0 & 0 & 0 & 0 \\
& k_{2,2} & 0 & 0 & 0 & k_{2,6} & 0 & 0 & k_{2,9} & 0 & 0 & 0 & k_{2,13} & 0 \\
& & k_{3,3} & 0 & k_{3,5} & 0 & 0 & 0 & 0 & k_{3,10} & 0 & k_{3,12} & 0 & 0 \\
& & & k_{4,4} & 0 & 0 & k_{4,7} & 0 & 0 & 0 & k_{4,11} & 0 & 0 & k_{4,14} \\
& & & & k_{5,5} & 0 & 0 & 0 & 0 & k_{5,10} & 0 & k_{5,12} & 0 & 0 \\
& S & & & & k_{6,6} & 0 & 0 & k_{6,9} & 0 & 0 & 0 & k_{6,13} & 0 \\
& & Y & & & & k_{7,7} & 0 & 0 & 0 & k_{7,11} & 0 & 0 & k_{7,14} \\
& & & M & & & & k_{8,8} & 0 & 0 & 0 & 0 & 0 & 0 \\
& & & & M & & & & k_{9,9} & 0 & 0 & 0 & k_{9,13} & 0 \\
& & & & E & & & & k_{10,10} & 0 & k_{10,12} & 0 & 0 \\
& & & & & T & & & & k_{11,11} & 0 & 0 & k_{11,14} \\
& & & & & & & R & & & & k_{12,12} & 0 & 0 \\
& & & & & & & & I & & & & k_{13,13} & 0 \\
& & & & & & & & & C & & & & k_{14,14}
\end{array}\right]
$$

The terms in (4) that refer to non-uniform torsion are given as [7] $k_{4,4}=k_{11,11}=-k_{4,11}=\frac{c k_{1}}{\kappa k_{2}}$, $k_{4,7}=k_{4,14}=-k_{7,11}=-k_{11,14}=-c=\frac{-k_{2}}{\kappa k_{2}^{2}-k_{1} k_{3}}, k_{7,7}=k_{14,14}=c\left(\kappa b_{1}-b_{0} k_{3} / k_{2}\right)$, and $k_{7,14}=c k_{3} / k_{2}$ , where $k_{1}=\frac{b_{1}}{E I_{\omega}}, k_{2}=\frac{b_{2}}{E I_{\omega}}$ and $k_{3}=\left(\frac{b_{3}}{E I_{\omega}}-\frac{b_{1}}{G I_{T s}}\right)$.

The effect of the secondary torsional moment is considered by means of the constant $\kappa=\left(1+\frac{I_{T}}{I_{T S}}\right)^{-1}$ and the transfer constants $b_{j}, j \in\langle 0,3\rangle$. If this effect is disregarded, $\kappa=1$. This is usually done for the case of beams with open cross-sections where the influence of the secondary torsional moment is insignificant [9]. However, for hollow cross-section (HCS) beams this effect must be considered, as was previously shown in [3] and [7]. The expression for the secondary torsion constant $I_{T}$ and $I_{T S}$ and $I_{\omega}$ depends on the chosen form of the crosssection [15]. The element stiffness matrix for non-uniform torsion was derived in [7] in the local coordinate system, using the transfer relations. The indices $m, n$ by the stiffness constants $k_{m, n}$ denote their position in the local stiffness matrix $\left[K^{l e}\right]$.

The terms in (4) that represent the obvious axial and flexural stiffness read

$$
\begin{aligned}
& k_{1,1}=k_{8,8}=-k_{1,8}=\frac{E A}{L}, k_{2,2}=k_{9,9}=-k_{2,9}=12 \frac{E I_{z}}{L^{3}}\left(1+12 \rho_{z}\right), k_{2,6}=k_{2,13}=6 \frac{E I_{z}}{L^{2}}\left(1+12 \rho_{z}\right), \\
& k_{3,3}=k_{10,10}=-k_{3,10}=12 \frac{E I_{y}}{L^{3}}\left(1+12 \rho_{y}\right), k_{3,5}=+k_{3,12}=-k_{10,12}=k_{5,10}=-6 \frac{E I_{y}}{L^{2}}\left(1+12 \rho_{y}\right),
\end{aligned}
$$




$$
\begin{aligned}
& k_{5,5}=k_{12,12}=\frac{E I_{y}}{L}\left(1+\frac{3}{1+12 \rho_{y}}\right), k_{6,6}=k_{13,13}=\frac{E I_{z}}{L}\left(1+\frac{3}{1+12 \rho_{z}}\right), \\
& k_{6,9}=k_{9,13}=-6 \frac{E I_{z}}{L^{2}}\left(1+12 \rho_{z}\right), k_{6,13}=\frac{E I_{z}}{L}\left(\frac{3}{1+12 \rho_{z}}-1\right) \text { and } k_{5,12}=\frac{E I_{y}}{L}\left(\frac{3}{1+12 \rho_{y}}-1\right) .
\end{aligned}
$$

Parameters $\rho_{y}=\frac{E I_{y}}{L^{2} k_{y}^{s} G A}$ and $\rho_{z}=\frac{E I_{z}}{L^{2} k_{z}^{s} G A}$ are stiffness ratios, with $k_{y}^{s}$ and $k_{z}^{s}$ denoting the shear correction factors.

The primary and the secondary torsional moment at the nodal points are given as [3]

$$
M_{T s i}=\kappa\left(M_{T i}-G I_{T} \vartheta_{M i}^{\prime}\right), \quad M_{T s k}=\kappa\left(M_{T k}-G I_{T} \vartheta_{M k}^{\prime}\right)
$$

and

$$
M_{T p i}=M_{T i}-M_{T s i}, \quad M_{T p k}=M_{T k}-M_{T s k}
$$

The expressions for the calculation of the axial stresses and the shear stresses depend on the type of the cross-sectional area, as was described in detail in [3]. These expressions will be used for calculation of the stresses in the numerical investigation in chapter 3.

\subsection{Transformation of the element stiffness matrix from local to global coordinates}

The element stiffness matrix (4), the displacement vector (1), and the load vector (2) have to be transformed from the local to the global coordinate system. The transformation is performed with the help of the extended transformation matrix.

The element stiffness matrix in the global coordinate system can formally be written as

$$
\left[K^{g e}\right]=\left[T^{e}\right]^{T}\left[K^{l e}\right]\left[T^{e}\right]
$$

where $\left[T^{e}\right]$ is the $14 \times 14$ transformation matrix for finite element $e$ and $\left[T^{e}\right]^{T}$ denotes its transpose.

Figure 2 shows the position of the local and global coordinate systems at a chosen nodal point, for the special case of $Z$ being parallel to $z$. The $x-y$ plane coincides with the $X-Y$ plane.

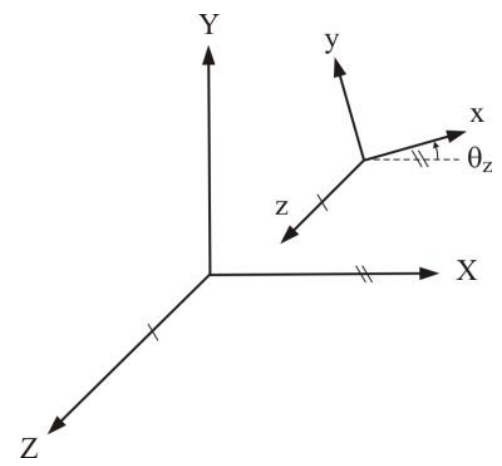

Fig. 2 Global and local coordinate system - rotation in the $X-Y$ plane, with $\theta_{z}$ as the angle of rotation.

The transformation submatrix $\left[T_{z}\right]$ is given as 


$$
\left[T_{z}\right]=\left[\begin{array}{ccccccc}
\cos \theta_{z} & \sin \theta_{z} & 0 & 0 & 0 & 0 & 0 \\
-\sin \theta_{z} & \cos \theta_{z} & 0 & 0 & 0 & 0 & 0 \\
0 & 0 & 1 & 0 & 0 & 0 & 0 \\
0 & 0 & 0 & \cos \theta_{z} & \sin \theta_{z} & 0 & 0 \\
0 & 0 & 0 & -\sin \theta_{z} & \cos \theta_{z} & 0 & 0 \\
0 & 0 & 0 & 0 & 0 & 1 & 0 \\
0 & 0 & 0 & 0 & 0 & 0 & 1
\end{array}\right]
$$

The transformation matrices $\left[T_{x}\right]$ and $\left[T_{y}\right]$ are obtained by analogy. The whole transformation matrix $\left[T^{e}\right]$ and the global system of equations for the beam structure is obtained in the usual way.

The transformation of the nodal degrees of freedom from local to global coordinates is carried out by means of

$$
\left\{a^{g}\right\}=\left[T^{e}\right] \cdot\left\{a^{l}\right\}
$$

where

$$
\left\{a^{g}\right\}=\left\{\begin{array}{lllllll}
u^{g} & v^{g} & w^{g} & \vartheta_{X}^{g} & \vartheta_{Y}^{g} & \vartheta_{Z}^{g} & \vartheta_{M}^{\prime} g
\end{array}\right\}^{T}
$$

is the global displacements vector, and

$$
\left\{a^{l}\right\}=\left\{\begin{array}{lllllll}
u^{l} & v^{l} & w^{l} & \vartheta_{x}^{l} & \vartheta_{y}^{l} & \vartheta_{z}^{l} & \left.\vartheta_{M}^{\prime}\right\}^{T}
\end{array}\right.
$$

is the local displacements vector.

The new degrees of freedom, $\vartheta_{M}^{\prime g}=\vartheta_{M}^{\prime}$, is considered to be a scalar value.

After the displacements calculation, the distribution of the internal forces and the displacements in the axial and the transverse direction can be determined. The local rotation angles and the torsional moments for the case of non-uniform torsion can be computed by means of the local transfer relations [7]. The new 3D Timoshenko finite beam element with torsional warping (WT-beam) was coded with the help of MATHEMATICA [16] and used in the following analyses.

\section{Numerical investigation and experimental measurement of the influence of non- uniform torsion}

In the following, results from numerical analyses of two non-aligned member steel frame ( $E=200 \mathrm{GPa}, v=0.3$ ) of rectangular hollow cross-section (RHC) has been examined (Fig. 3). The beams have length $L_{1}=500 \mathrm{~mm}$ and $L_{2}=1000 \mathrm{~mm}$ and are connected perpendicularly at node $B$. The frame is clamped at cross-section at point $C$ and is subjected to a vertical force $F_{y}=-77,4 \mathrm{~N}$ at point $A$. The cross-sectional dimensions are: $t_{f}=t_{w}=2 \mathrm{~mm} ; b=18 \mathrm{~mm}$ and $h=58 \mathrm{~mm}$. The global coordinate system $X, Y, Z$ has the beginning at point $\mathrm{B}$. 


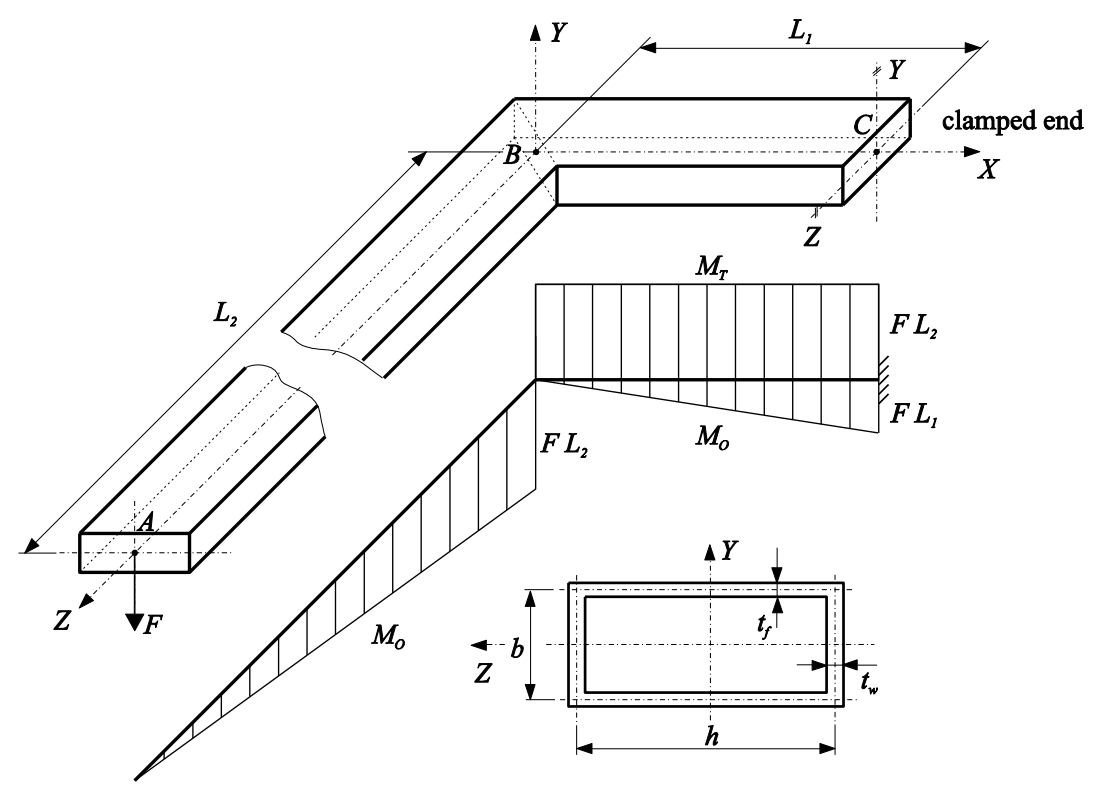

Fig. 3 Geometry, boundary conditions, loading and cross-section of the frame.

\subsection{Description of the cross-section characteristics}

The RHS cross-section parameters calculated by TTT [15] are in Table 1:

\begin{tabular}{|c|c|c|}
\hline Title & Expression & Value \\
\hline Cross-sectional area & $A=2\left(h t_{w}+b t_{f}\right)$ & $\begin{array}{l}304.0 \\
{\left[\mathrm{~mm}^{2}\right]}\end{array}$ \\
\hline Auxiliary constant & $\gamma=h / t_{w}+b / t_{f}$ & 38.0 \\
\hline $\begin{array}{l}\text { Warping ordinate at the } \\
\text { corners }\end{array}$ & $\omega_{R}=\frac{h b}{4} \cdot \frac{h t_{f}-b t_{w}}{h t_{f}+b t_{w}}$ & $\begin{array}{l}137.4 \\
{\left[\mathrm{~mm}^{2}\right]}\end{array}$ \\
\hline Warping constant & $I_{\omega}=\omega_{R}^{2} \frac{A}{3}$ & $\begin{array}{l}1.912 \times 10^{6} \\
{\left[\mathrm{~mm}^{6}\right]}\end{array}$ \\
\hline Torsion constant & $I_{T}=\frac{2(h b)^{2}}{\gamma}$ & $57365.0\left[\mathrm{~mm}^{4}\right]$ \\
\hline $\begin{array}{l}\text { Secondary torsion } \\
\text { constant }\end{array}$ & $I_{T s}=\frac{20 \gamma I_{\omega} A}{\frac{(A h b)^{2}}{h b t_{w} t_{f}}+\frac{\left(h^{2}+b^{2}\right)^{2}}{1,5}}$ & $\begin{array}{c}14589.3 \\
{\left[\mathrm{~mm}^{4}\right]}\end{array}$ \\
\hline $\begin{array}{l}\text { Quadratic moment of } \\
\text { area }\end{array}$ & $I=\frac{(b+t)^{3}(h+t)}{12}-\frac{(b-t)^{3}(h-t)}{12}$ & $\begin{array}{c}20885.3 \\
{\left[\mathrm{~mm}^{4}\right]}\end{array}$ \\
\hline $\begin{array}{l}\text { Intermediate cross- } \\
\text { sectional area }\end{array}$ & $A_{s}=b h$ & $\begin{array}{c}1044 \\
{\left[\mathrm{~mm}^{2}\right]}\end{array}$ \\
\hline
\end{tabular}

Tab. 1 General expressions for cross-sectional characteristics of RHS.

The shear correction factors are: $k_{z}^{s}=0.715, k=k_{y}^{s}=0.1337$ [5].

\subsection{Solution of internal moments and deformation}

The elastostatic analysis of the frame is done by number of 200 of the WT beam finite elements with following results: 
Distribution of the bending and torsional moment, $M_{o}$ and $M_{T}$, is shown in Figure 3. Distribution of the bimoment, $M_{\omega}$, along the aligned centerline $C-B-A$ of the beam is shown in Figure $4\left(L=L_{1}+L_{2}\right)$.

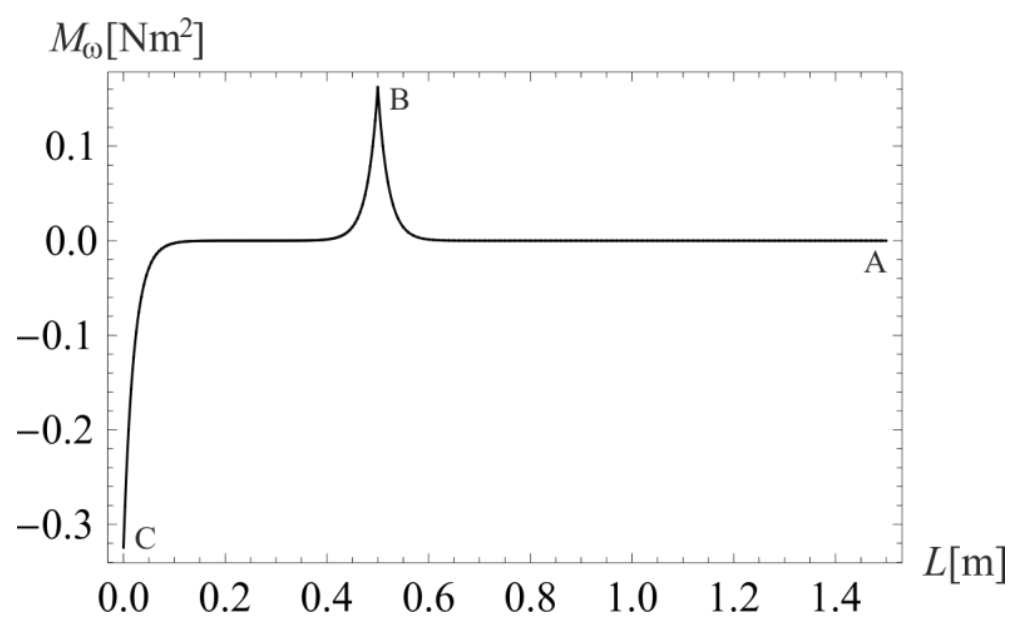

Fig. 4 Distribution of the bimoment.

Distribution of the primary, $M_{T p}$, and secondary, $M_{T s}$, torsional moment along the aligned centerline $C-B-A$ is shown in Figure 5.

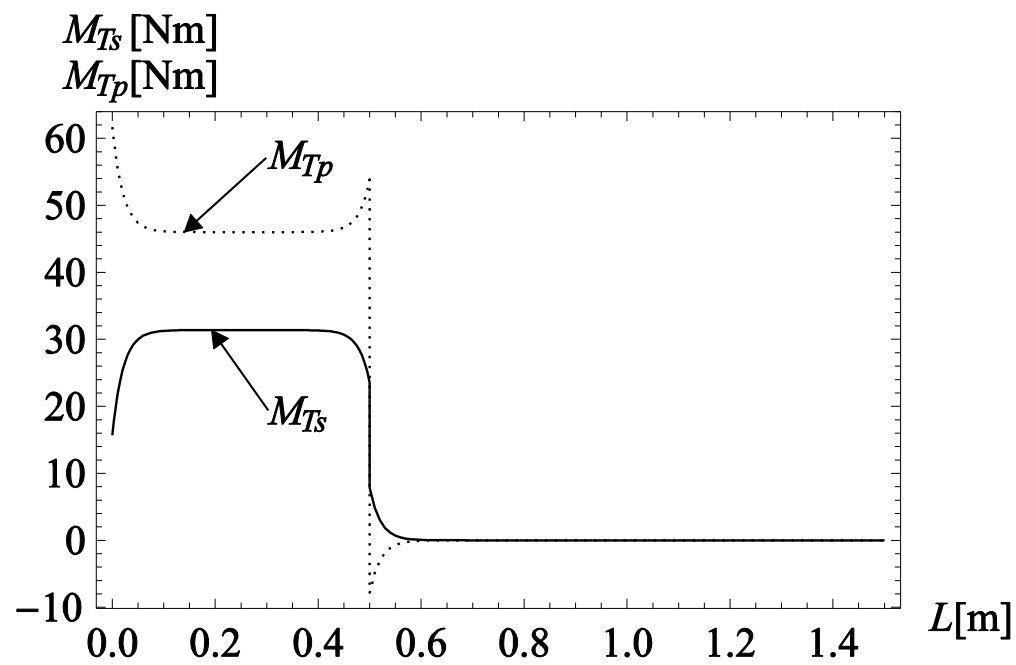

Fig. 5 Distribution of the primary and secondary torsional moment.

Numerical values of the internal moments at points $A, B$ and $C$ are in Tab. 2. The notation $B A$ means a position of the cross-section cut to the left of the point $B$, and $B C$ means the position to the right of the point $B$.

\begin{tabular}{|c|c|c|c|c|c|}
\hline Point & $\begin{array}{c}M_{T p} \\
{[\mathrm{Nm}]}\end{array}$ & $\begin{array}{c}M_{T s} \\
{[\mathrm{Nm}]}\end{array}$ & $\begin{array}{c}M_{T} \\
{[\mathrm{Nm}]}\end{array}$ & $\begin{array}{c}M_{o} \\
{[\mathrm{Nm}]}\end{array}$ & $\begin{array}{c}M_{\omega} \\
{\left[\mathrm{Nm}^{2}\right]}\end{array}$ \\
\hline$A$ & 0.00 & 0.00 & 0.00 & 0.00 & 0.00 \\
\hline$B A$ & -7.84 & 7.84 & 0.00 & 77.39 & 0.162 \\
\hline$B C$ & 53.85 & 23.54 & 77.39 & 0.00 & 0.162 \\
\hline$C$ & 61.70 & 15.69 & 77.39 & 38.69 & -0.324 \\
\hline
\end{tabular}

Tab. 2 Internal moments and bimoment at relevant points of the frame.

Deformed configuration of the frame is shown in Figure 6. Numerical values of the relevant global displacements at the points $B$ and $A$ (according the notation (10)) are listed in Table 3 . 


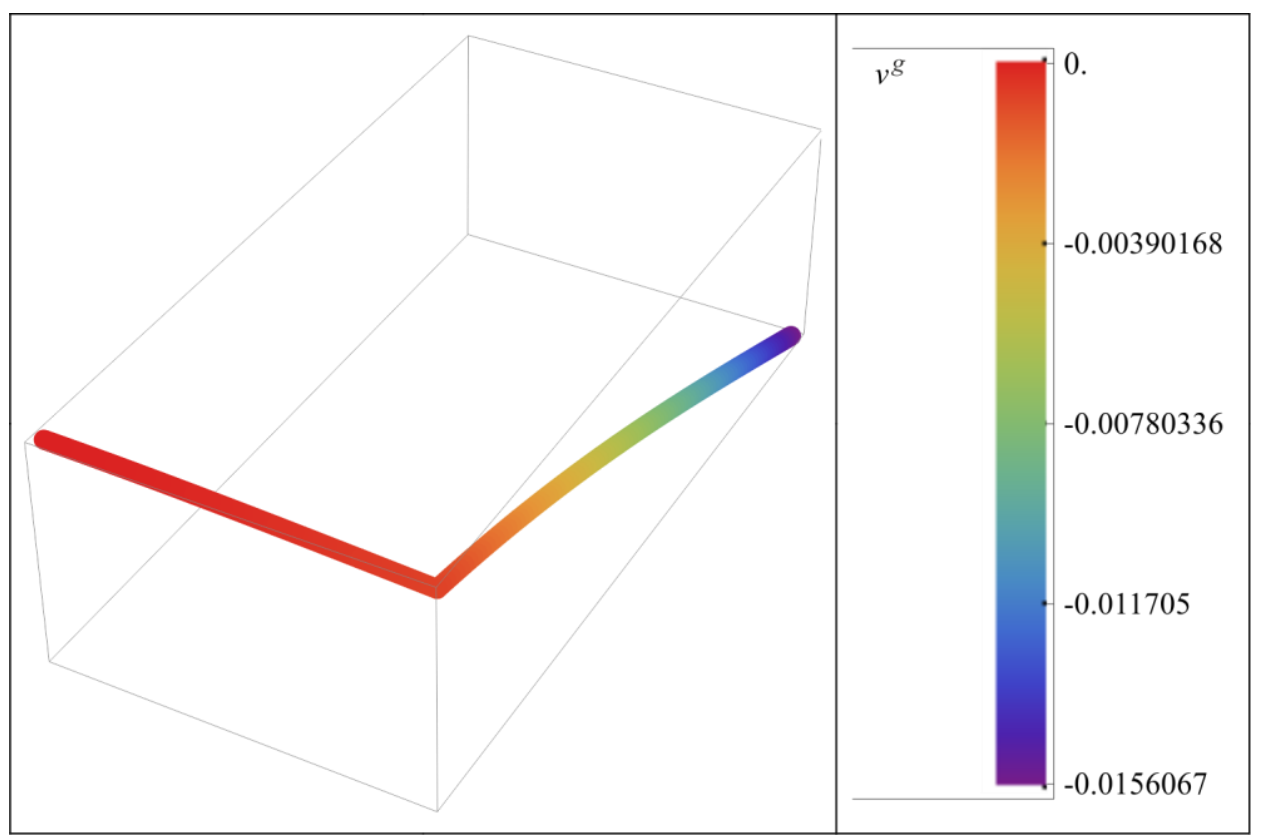

Fig. 6 Deformation of the frame.

\begin{tabular}{|c|c|c|c|c|}
\hline Point & $v^{g}[\mathrm{~mm}]$ & $\vartheta_{X}^{g}[\mathrm{rad}]$ & $\vartheta_{Z}^{g}[\mathrm{rad}]$ & $\vartheta_{M}^{\prime g}[\mathrm{rad} / \mathrm{m}]$ \\
\hline$B$ & -0.78 & -0.0086 & 0.00231 & 0.00877 \\
\hline$A$ & -15.64 & -0.018 & 0.00228 & 0.0 \\
\hline
\end{tabular}

Tab. 3 Global displacements at the points B and A.

The displacement at point $A$ is calculated analytically by the Castigliano's first theorem: $v_{A}^{g}=\frac{\partial A_{\sigma \tau}}{\partial F}$; where $A_{\sigma \tau}=A_{\sigma}+A_{\tau 1}+A_{\tau 2}$ is the total stress energy in the frame. There, $A_{\sigma}=\frac{1}{2 E} \int_{V} \sigma_{o}^{2} d V$ is the normal stress energy due to bending moment, $A_{\tau 1}=\frac{1}{2 G} \int_{V} \tau_{1}^{2} d V$ is the shear stress energy due to torsional moment, $A_{\tau 2}=\frac{1}{2 G} \int_{V} \tau_{2}^{2} d V$ is the shear stress energy due to shear force, and $V$ is the volume of the frame. After some mathematical operations, the vertical displacement at the end $A$ is:

$v_{A}^{g}=-\left(\frac{F}{3 E I}\left(L_{1}^{3}+L_{2}^{3}\right)+\frac{F L_{2} A}{G}\left(\frac{L_{1}}{2 A_{s} t}\right)^{2}+\frac{F\left(L_{1}+L_{2}\right)}{G A k^{2}}\right)=-(6.95+8.77+0.28)=-15.99[\mathrm{~mm}]$ that consists of parts due to bending and uniform torsion with Bredt's stress, and shear forces with the shear correction factor $k=k_{y}^{s}=0.1337$ [5]. The shear modulus is $G=\frac{E}{2.6}=76923$ $\mathrm{MPa}$. The analytical solution with uniform torsion gives comparable displacement with this obtained by our warping torsion method (see Table 3).

\subsection{Normal and shear stress calculation in the cross-section at point $C$}

As listed in Table 2, the cross-section at point $C$ (Figure 7) is loaded by the bending moment, bimoment, primary and secondary torsional moment. 


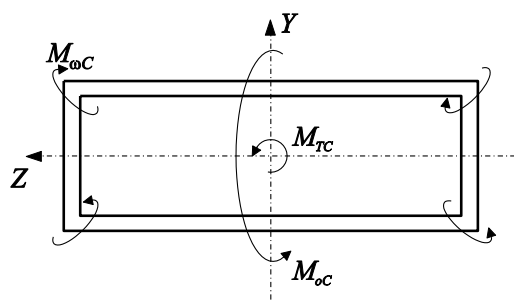

Fig. 7 Internal moments in cross at point $C$.

The bending normal stress is: $\sigma_{o C}=\frac{M_{o C}}{I_{z}} y$, where the quadratic moment of area is: $I_{z}=20885.3 \mathrm{~mm}^{4}$. The position $y$ is: $y \in<y_{\max }, y_{\min }>$ with $y_{\max }= \pm\left(b+t_{w}\right) / 2= \pm 10 \mathrm{~mm}$, and $y_{\min }= \pm\left(b-t_{w}\right) / 2= \pm 8 \mathrm{~mm}$. Then, $\sigma_{o C \max }= \pm 18.53 \mathrm{MPa}$ and $\sigma_{o C \min }= \pm 14.82 \mathrm{MPa}$. The average bending stress in the middle of the thickness $t$ is $\sigma_{o \text { Cav }}= \pm 16.67 \mathrm{MPa}$.

The bimoment normal stress in the corners of the cross-section is:

$\sigma_{R C}= \pm \frac{M_{\omega C}}{I_{\omega}} \omega_{R}= \pm 23.27 \mathrm{MPa}$.

After superposition of the maximal bending stress and bimoment normal stress in the corners, the resultant, maximal and minimal normal stress is obtained: $\sigma_{r C \max }= \pm \sigma_{o C \max } \pm \sigma_{R C}= \pm 41.79 \mathrm{MPa}, \sigma_{r C \text { min }}= \pm \sigma_{o C \max } \mp \sigma_{R C}=\mp 4.74 \mathrm{MPa}$.

After superposition of the average bending stress and the bimoment normal stress in the corners, the average resultant, maximal and minimal, normal stress is obtained: $\sigma_{r \text { Cav } \max }= \pm \sigma_{o \text { Cav }} \pm \sigma_{R C}= \pm 39.94 \mathrm{MPa}, \sigma_{r \text { Cav min }}= \pm \sigma_{o \text { Cav }} \mp \sigma_{R C}=\mp 6.6 \mathrm{MPa}$.

Distribution of the resultant, maximal and minimal normal stress is depicted in Figure 8. 

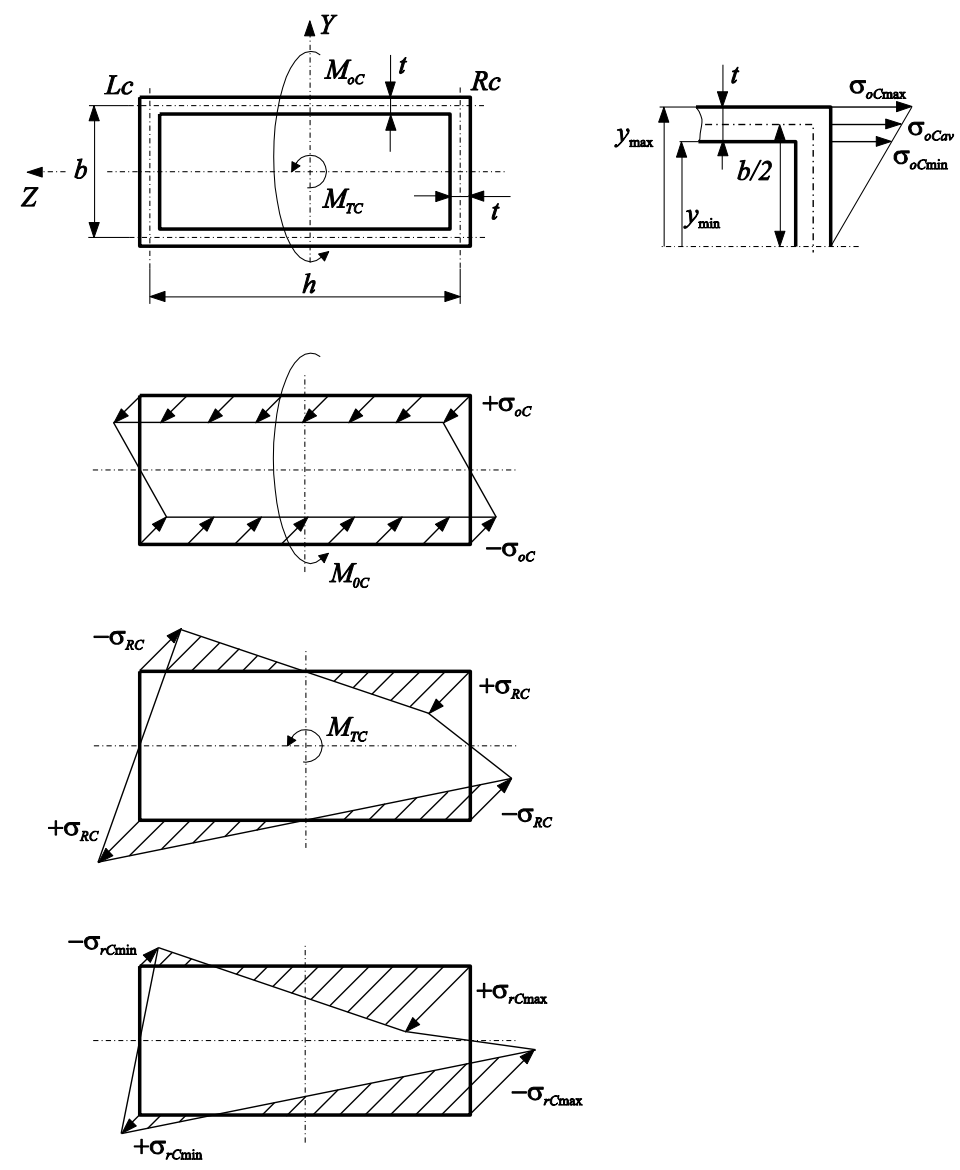

Fig. 8 The normal stress distribution in the cross-section at the point $C$.

The torsional shear stress in non-uniform torsion theory including STMDE is divided into two components, namely on a stress caused by the primary and the secondary torsional moment [3].

The primary shear flow at point $C$ is: $T_{p C} \frac{M_{T p C}}{2 b h}=29.54 \mathrm{~N} / \mathrm{mm}$. Because of constant thickness of whole cross-section, $t_{f}=t_{w}=t$, the primary torsional shear stress is constant along the circumference of the cross section: $\tau_{p C}=\frac{T_{p C}}{t}=14.77 \mathrm{MPa}$ (Figure 9a).

The secondary shear flow and the secondary torsional shear stress varies along the circumference of the cross section [3].

The limit values of the secondary shear flow at points $0,1,2$ of the cross-section (Figure 9) read: $T_{0, s C}=\frac{M_{T S C}}{I_{\omega}} S_{0}=15.03 \mathrm{~N} / \mathrm{mm} ; T_{1, s C}=\frac{M_{T S C}}{I_{\omega}} S_{1}=21.17 \mathrm{~N} / \mathrm{mm}$;

$T_{2, s C}=\frac{M_{T s C}}{I_{\omega}} S_{2}=-17.66 \mathrm{~N} / \mathrm{mm}$.

There, $S_{0}=\frac{h^{2}-b^{2}}{6(h / t+b / t)} \omega_{R}=1831.6 \mathrm{~mm}^{2}, S_{1}=S_{0}+A_{f} \frac{\omega_{R}}{4}=3067.9 \mathrm{~mm}^{2}$, 
$S_{2}=S_{0}-A_{w} \frac{\omega_{R}}{4}=2452.1 \mathrm{~mm}^{2}$ are the auxiliary constants, and $A_{f}=b t=36 \mathrm{~mm}^{2}$ is the area of the flange and $A_{w}=h t=116 \mathrm{~mm}^{2}$ is the area of the web.

The limit values of the secondary torsional shear stress at point $C$ read (Figure 9b): $\tau_{0, s C}=\frac{T_{0, s C}}{t}=7.51 \mathrm{MPa}, \tau_{1, s C}=\frac{T_{1, s C}}{t}=12.59 \mathrm{MPa}, \tau_{2, s C}=\frac{T_{2, s C}}{t}=-8.83 \mathrm{MPa}$.

Resultant torsional shear stress in the corners of the cross-sectional area at $C$ is: $\tau_{0 C}=\tau_{p C}+\tau_{0, s C}=22.29 \mathrm{MPa}$. If the torsional shear stress is calculated by Bredt's formula, the torsional shear stress is: $\tau_{C}=\frac{M_{T C}}{2 h b t}=18.53 \mathrm{MPa}$. As can be shown, the shear stress due to shear force is not significant and therefore neglected.

a)

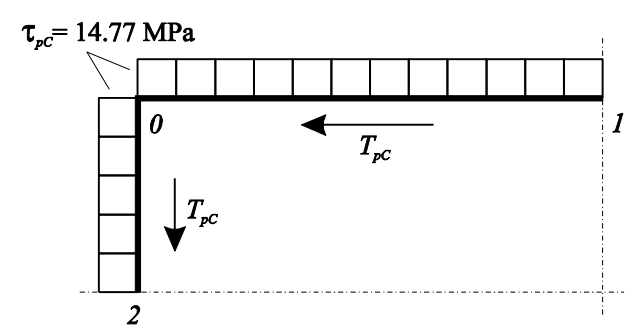

b)

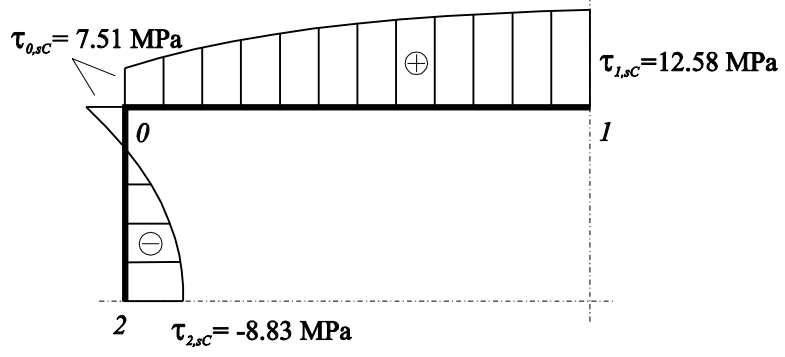

Fig. 9 Distribution of the torsional shear stress in the cross-section symmetric part at the point $C$.

Maximal von Mises stress is in the left corners (Rc, Figure 3) is:

$\sigma_{R c}=\sqrt{\sigma_{r C \max }^{2}+3 \tau_{0 C}^{2}}=56.9 \mathrm{MPa}$.

The von Mises stress in the right corners ( $L c$, Figure 3) is:

$\sigma_{L c}=\sqrt{\sigma_{r C \min }^{2}+3 \tau_{0 C}^{2}}=38.9 \mathrm{MPa}$.

If both the bimoment normal stress and the STDME are neglected (Saint Venant torsion), the maximal von Mises stress is: $\sigma_{S V}=\sqrt{\sigma_{o C \max }^{2}+3 \tau_{C}^{2}}=19.97 \mathrm{MPa}$. Comparison of the von Mises stress shows that neglecting of warping and STMDE may lead to significant underestimation of the frame.

\section{Verification of the solution results obtained by the WT warping beam finite element}

The same example as studied in previous chapter is solved by ANSYS [5] using the BEAM188 (warping restrained, WR) and warping unrestrained, WU) and SHEL181 and SOLID186 finite elements. The rectangular hollow cross-section of the frame is considered by the finite element models. Measurements are performed for confirmation of the calculated results. Figure 10 shows the measured frame assembly. It should be noted that in contrast to the calculation models where the rectangular hollow cross-section of the beams was considered, the measured frame, given the practical possibilities, is made of two beams of hollow crosssection with rounded corners (Figure 11) with $r=2 \mathrm{~mm}$. 


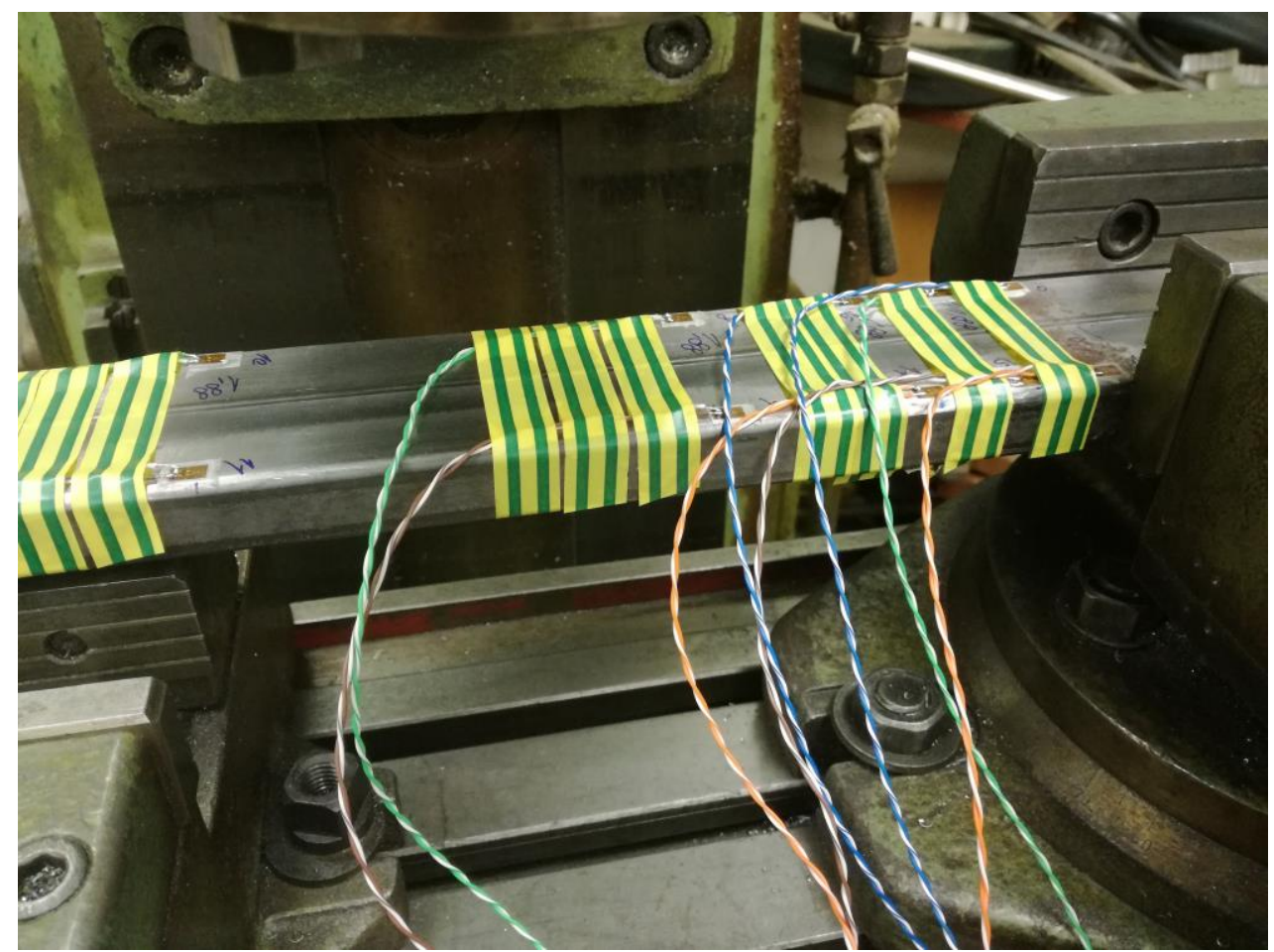

Fig. 10 Measured frame assembly.

The deflection of the frame was measured by micrometer in the points $A$ and $B$. Normal stress due to bending moments and bimoment is measured by tensometers (strain gauges). Their position on the twisted part of the frame is shown in Figure 11 and Table 4. They are positioned at the places where the maximal influence of the non-uniform torsion has been shown in the above calculations. Due to strain gauge measurements, it was not possible to place the strain gauges on profile edges or directly at the clamped end $C$. The mechatronic set-up included a set of tensometers (type HBM 1,5/120LY61), strain/bridge input measuring module NI-9235 (8 differential analog input channels, bridge resistance $120 \Omega$ ), and a laptop with software LabVIEW for acquisition of measurement data.

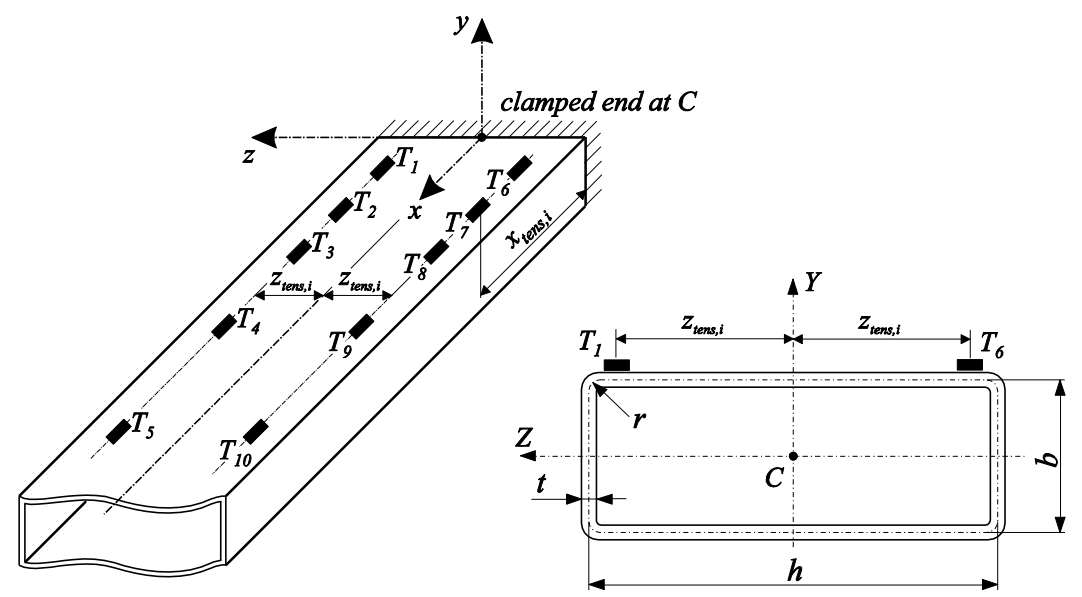

Fig. 11 Position of the tensometers on the twisted part of the frame and the cross-section.

\begin{tabular}{|c|c|c|c|c|c|}
\hline Tensometers & $T_{1,6}$ & $T_{2,7}$ & $T_{3,8}$ & $T_{4,9}$ & $T_{5,10}$ \\
\hline$x_{\text {tens }, i}[\mathrm{~mm}]$ & 3 & 25 & 50 & 100 & 220 \\
\hline$z_{\text {tens }, i}[\mathrm{~mm}]$ & \multicolumn{5}{|c|}{ \pm 24} \\
\hline
\end{tabular}

Tab. 4 The position of the tensometers $T_{i}$ is defined by coordinates $x_{\text {tens, } i}$ and $z_{\text {tens }, i}$. 
Comparison of the deflection at the points $A$ and $B$, obtained by WT and BEAM188 (WU and WR) and SHELL181 and Solid186 finite elements, and by measurement (M), is shown in Table 5.

\begin{tabular}{|c|c|c|c|c|c|c|}
\hline $\begin{array}{c}\text { Number of } \\
\text { FE }\end{array}$ & WT & $\begin{array}{c}\text { SHELL181 } \\
44394\end{array}$ & $\begin{array}{c}\text { SOLID 186 } \\
65520\end{array}$ & $\begin{array}{c}\text { WR } \\
150\end{array}$ & WU & M \\
\hline$v_{A}^{g}[\mathrm{~mm}]$ & -15.64 & -18.80 & -17.17 & -15.43 & -15.50 & -17.20 \\
\hline$v_{B}^{g}[\mathrm{~mm}]$ & -0.78 & -0.85 & -0.88 & -0.78 & -0.78 & -0.97 \\
\hline
\end{tabular}

Tab. 5 Comparison of the calculated and measured vertical displacements.

As it has been shown before, the analytical solution by the Castigliano's first theorem results the displacement at point $A: v_{A}^{g}=-15.99 \mathrm{~mm}$.

Table 5 shows that all the beam theories produce smaller displacements compared to the SHELL181 and SOLID186 solution, and to the measurement results as well. It follows from that point of view that the beam theories solutions are less accurate compared to the measurement, but the difference in the displacements is not significant. As shown in [14], the difference is caused most likely by the torsional distortion of the thin-walled hollow crosssections, what is not considered in the above calculations done by the beam finite elements. On the other side, a preparation of the computational model of the spatial beam structures through the shell and solid finite elements is quite demanding. From this point of view, the consideration of the Saint Venant torsion theory could be assumed as satisfactorily for the displacement calculation in the solved example. However, as shown in [3],[7],[12],[13] and also in the following investigations, the warping torsion effect and the STDME influence the stress state significantly and therefore could not be neglected.

The above normal stress calculated numerically by the WT beam finite elements is verified using the SHELL181 and SOLID186 finite elements [5]. Normal stress has been also measured at the positions from Table 4 and Figure 11 on the twisted part of the frame made of rounded hollow cross-section beams. Measured values of the resultant normal stress, $\sigma_{r m}$, due to bending moment and bimoment is presented in Table 6 .

\begin{tabular}{|c|c|c|c|c|c|c|c|c|c|c|}
\hline Tensometer & $T_{1}$ & $T_{2}$ & $T_{3}$ & $T_{4}$ & $T_{5}$ & $T_{6}$ & $T_{7}$ & $T_{8}$ & $T_{9}$ & $T_{10}$ \\
\hline$\sigma_{r m}[\mathrm{MPa}]$ & 11.5 & 12.5 & 13.2 & 14.4 & 10.4 & 30.5 & 23.2 & 18.1 & 12.9 & 8.9 \\
\hline$\sigma_{o m}[\mathrm{MPa}]$ & 20.9 & 18.2 & 16.4 & 14.8 & 10.1 & 20.9 & 18.7 & 16.4 & 14.8 & 10.2 \\
\hline$\sigma_{\omega m}[\mathrm{MPa}]$ & -9.4 & -5.7 & -3.2 & -0.4 & 0.3 & 9.6 & 4.5 & 1.7 & -1.9 & -1.3 \\
\hline
\end{tabular}

Tab. 6 Normal stress obtained by the measurement.

By subtraction, resp. by addition the measured normal stress caused by bending, $\sigma_{\text {om }}$, from the resultant normal stress $\sigma_{r m}$, the normal stress due to bimoment, $\sigma_{\omega m}$, is obtained. Their values are shown in Table 6. A certain disproportion of the measurement results in Table 6 is due to the implementation conditions and accuracy of the tensometric measurement itself. Nevertheless, the measured results can be considered as valid.

Distribution of the bimoment normal stress $\sigma_{\omega}$ (obtained with WT beam finite element) along the right upper edge of the twisted part $C-B$ of the frame is presented in Figure 12 ( $L=L_{1}+L_{2}$ ) together with the stress values obtained from measurement by the tensometers $T_{6}-T_{10}$ (marked by the black dots). As shown in Figure 12, a good agreement of the calculated and measured results is obtained. 


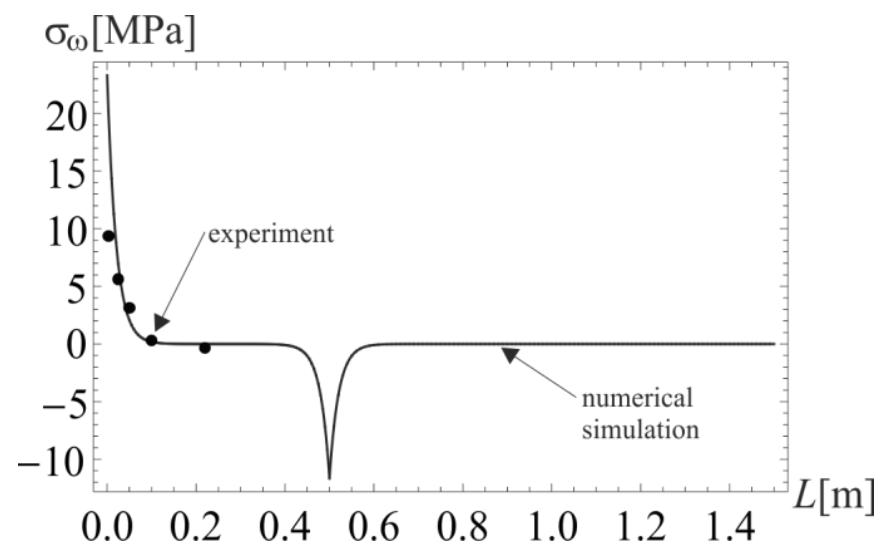

Fig. 12 Distribution of the bimoment normal stress $\sigma_{\omega}$ obtained by measurement and WT.

The resultant normal stress $\sigma_{r c}$ comparison, calculated by SHELL181 and SOLID186 finite elements [5], along the right upper edge of the twisted part $C-B$ of the frame is shown in Figure $13\left(L=L_{1}+L_{2}\right)$ together with the stress values (marked by black dots) measured by the tensometers $T_{6}-T_{10}$.

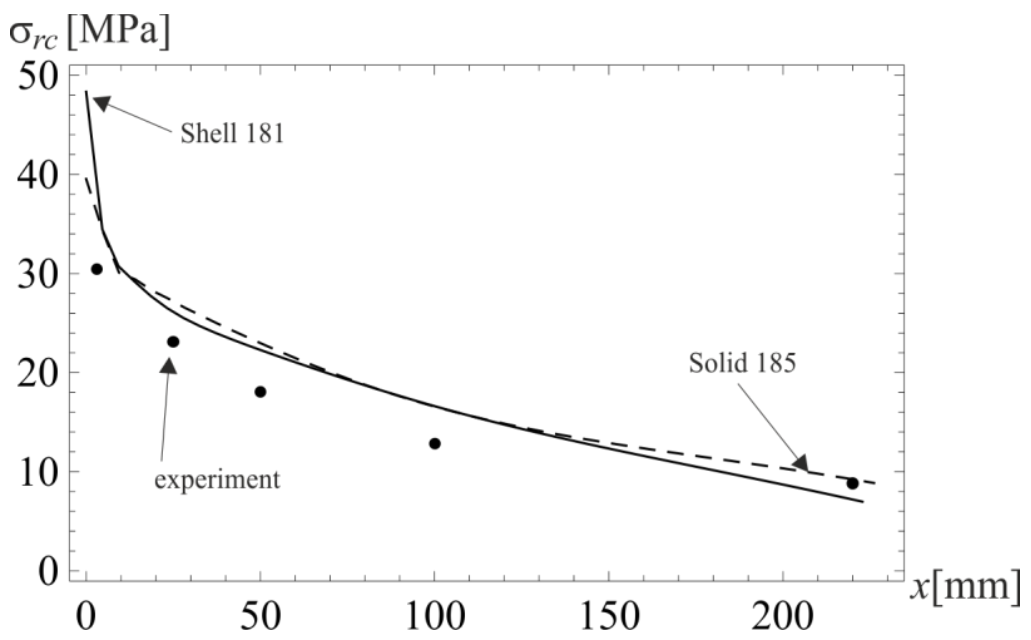

Fig. 13 Comparison of the calculated resultant normal stress $\sigma_{r c}$ with the measurement.

The above comparison shows that the resultant normal stress $\sigma_{r c}$ increases very strong to its maximal values in the corner $R_{c}$ which are listed in the Table 7.

\begin{tabular}{|c|c|c|c|}
\hline Finite element & SHELL181 & SOLID186 & WT \\
\hline $\begin{array}{c}\text { Maximal resultant normal } \\
\text { stress [MPa] at } R_{c}\end{array}$ & 48.3 & 39.5 & 41.79 \\
\hline
\end{tabular}

Tab. 7 Comparison of the maximal resultant stress in the corner $R c$.

The difference in the maximal normal stress (Figure 13) between the solid and shell finite element solution results from different properties of the elements and the effect of the boundary condition. Our results obtained with the WT finite element is located between the both results. The trend of the steep increase in the measured normal stress corresponds to the trend of the calculated stress, but its values, in particular due to the location of the strain gauges and the rounding of the corners, are smaller, which is understandable.

The comparison of the resultant normal stress $\sigma_{r c t}$ calculated by SHELL181 and SOLID186 finite elements [5], along the line allocated by $z_{\text {tens }, i}=24 \mathrm{~mm}$ (Figure 11) from the $x$ axis is 
shown in Figure 14, together with the stress values (marked by black dots) measured by the tensometers $T_{6}-T_{10}$. Maximal calculated values of the stress are given in Table 8 .

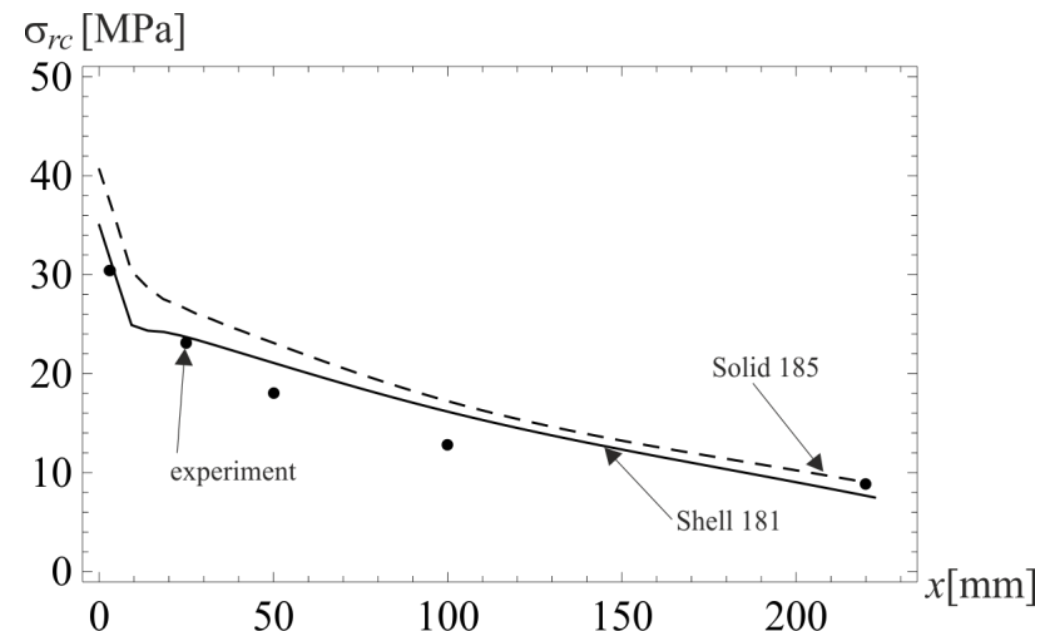

Fig. 14 Comparison of the resultant calculated normal stress $\sigma_{r c t}$ with the measurement on the shifted line.

\begin{tabular}{|c|c|c|}
\hline Finite element & SHELL181 & SOLID186 \\
\hline $\begin{array}{c}\text { Maximal resultant normal } \\
\sigma_{r c t} \text { stress }[\mathrm{MPa}]\end{array}$ & 35.0 & 40.6 \\
\hline
\end{tabular}

Tab. 8 Maximal calculated values of the resultant normal stress from the Figure 14.

The results in Figure 13 and Figure 14 and Table 6 and Table 7 verify not only correctness of the measured results but also acceptable accuracy of our WT finite element (Figure 12).

\section{SUMMARY AND CONCLUSIONS}

This paper has dealt with elastostatic analysis of the spatial frame consisted of two angled members of the rectangular hollow cross-section (RHS). The 3D Timoshenko beam (WT) finite element [7] of open and closed cross-sections, considering non-uniform torsion and STDME, is used in the analysis. The frame is loaded by bending and torsion. Obtained results concerning deformation and stress are compared with the ones obtained from solution by the standard beam, shell and solid finite elements [5]. Verification of the numerical results is done by the measurements.

The main contribution of the article is the application of our effective 3D Timoshenko beam finite element with torsional warping to the elastostatic analysis of angled beams with consideration of non-uniform torsion. It should be noted that in literature, except [14], can find research articles that deal with the non-uniform torsion of only straight and curved beams. While standard software contain the beam finite elements considered warping, but their application is only relevant to open profile beams, whereby the bimoment and bimoment stresses evaluation is inconvenient especially for closed profiles. The influence of the secondary torsion moment deformation effect, which is especially important for the closed profiles, is not reflected. It should also be noted that the classical analytical methods, intended for solution of non-uniform torsion, can only be applied to straight, generally of an single thin-walled beams. Such a task can also be solved by the shell and solid finite elements, but their application to the spatial beam structures is rather complicated and inefficient.

The results of the comparative calculations as well as the results of the experimental measurement confirmed the high efficiency and good precision of our end beam finite element. 
It is also shown that neglecting the effects of warping upon analysis of the beams with a thinwalled closed cross section leads to a great under-estimation of the bearing capacity of such a system. On the other hand, it has been shown that the relevant deformation of the system can also be obtained by applying the Saint Venant theory of torsion. It may also have led to claim in the Eurocode 3 [17] that in design of thin walled steel structures of the closed cross-section the influence of non-uniform torsion could be neglected.

\section{ACKNOWLEDGEMENT}

The research on which this paper is based was supported by the Grant Agency VEGA (grant No.: 1/0102/18 and 1/0081/18). These VEGA grants are developed based on the APVV -024612 project solution.

\section{REFERENCES}

[1] V. Z. Vlasov. Thin-walled elastic beams. National Science Foundation, Washington, 1961.

[2] K. Roik, G. Sedlacek. Theorie der Wölbkrafttorsion unter Berücksichtigung der sekundären Schubverformungen - Analogiebetrachtung zur Berechnung des querbelasteten Zugstabes. Stahlbau 1966; 35, 43.

[3] H. Rubin. Wölbkrafttorsion von Durchlaufträgern mit konstantem Querschnitt unter Berücksichtigung sekundärer Schubverformung. Stahlbau 2005; 74, Heft 11, 826.

[4] EN 1993 - Eurocode 3: Design of steel structures, European Committee for Standardization (CEN) 2004.

[5] ANSYS Swanson Analysis System, Inc., 201 Johnson Road, Houston, PA 15342/1300, USA.

[6] RSTAB, Ingenieur - Software Dlubal GmbH, Tiefenbach 2006.

[7] J. Murin, M. Aminbaghai, V. Kutis, V. Kralovic, V. Goga, HA. Mang. A new 3D Timoshenko finite beam element including non-uniform torsion of open and closed cross sections. Eng Struct 2014 (59), 153 - 160.

[8] IC Dikaros, EJ Sapountzakis, AK Argyridi. Generalized warping effect in the dynamic analysis of beams of arbitrary cross section. J Sound Vib 2016 (369), 119 - 146.

[9] M. Aminbaghai, J. Murin, J. Hrabovsky, HA. Mang. Torsional warping eigenmodes including the effect of the secondary torsion moment on the deformations. Eng Struct 2016 (106), 299 - 316.

[10] IC Dikaros, EJ Sapountzakis, AK Argyridi. Generalized warping effect in the dynamic analysis of beams of arbitrary cross section. J Sound Vib 2016 (369), 119 - 146.

[11] IN Tsipsis, EJ Sapountzakis. Generalized warping and distortional analysis of curved baems with isogeometric methods. Comp and Struct 2017 (191), 33 - 50.

[12] J. Murin, V. Goga, M. Aminbaghai, J. Hrabovsky, T. Sedlar, HA. Mang. Measurement and modelling of torsional warping free vibrations of beams with rectangular hollow cross-sections. Eng Struct 2017 (136), 68 - 76.

[13] M. Aminbaghai, J. Murin, G. Balduzzi, J. Hrabovsky, G. Hochreiner, HA. Mang. Secondorder torsional warping theory considering the secondary torsion-moment deformationeffect. Eng Struct 2017 (147), 724 - 739. 
[14] IC Dikaros. Advanced beam theories for the analysis of beam structures. PhD Dissertation. School of Civil Engineering. National Technical University of Athens. Greece, 2016, pp. 379.

[15] H. Rubin. Torsions-Querschnittswerte für rechteckige Hohlprofile nach EN 10210-2: 2006. Stahlbau, 76, Heft 1, 2007.

[16] Wolfram Research, Inc., MATHEMATICA, Version 8.0, Champaign, IL, USA, 2010.

[17] EN 1993 - Eurocode 3: Design of steel structures, European Committee for Standardization (CEN) 2004. 